\title{
Drug-Related Cardiotoxicity for the Treatment of Haematological Malignancies in Elderly
}

\author{
Alessandra Malato, Giorgia Saccullo, Giovanni Fazio, Beatrice Vergara, Simona Raso, Giovanni Paolo \\ Guarneri, Antonio Russo, Vincenzo Abbadessa and Sergio Siragusa*
}

Cattedra ed U. O. di Ematologia con trapianto, Azienda Ospedaliera Universitaria Policlinico di Palermo, Via del Vespro 129, 90127 Palermo

\begin{abstract}
Several publications have focused on the cardiotoxicity of specific classes of haematological therapeutic agents such as antracyclines and cyclofosfamide. Cardiotoxicity of cancer chemotherapeutics is a problem for patients of all ages, but it increases with age. Toxicity can also be developed months after the last chemotherapy dose, and late reactions can be seen years later when they present new-onset cardiomyopathy. No data are available about the cardiotoxicity of non-chemotherapy agents currently used as preferred therapy for haematological malignancy in elderly. In this review we have provided a summary of the cardiovascular toxic effects produced by different drugs and therapeutic agents. Early identification of patients who are at risk for cardiotoxicity should be a primary goal for haematologists in the development of personalised antineoplastic therapeutic strategies or interventions. Thus, the discovery of new biomarkers to identify patients at a high risk for the development of these complications is a high priority. Although targeted therapies such as imatinib and anti-CD20 antibody, such as rituximab, are considered less toxic and better tolerated by patients compared with classic chemotherapy drugs, certain cardiological complications can be very serious as these agents have been in use for a limited period of time.
\end{abstract}

Keywords: Haematological malignancies, cardiotoxicity, heart failure, imatinib, rituximab, antracyclines, cyclofosfamide.

\section{INTRODUCTION}

Cardiotoxicity is a frequent side effect of chemotherapeutic agents used in haematological malignancies. Chemotherapyassociated cardiotoxicity varies from transient blood pressure and/or electrocardiographic (EKG) changes to more serious events such as arrhythmias, myocarditis, pericarditis, myocardial infarction and cardiomyopathy, which may end in left ventricular dysfunction (LVD) or congestive heart failure (CHF) [1]

Since cardiotoxicity is often dose-dependent, it has a serious impact on treatment efficacy; cardiac complications of treatment lead to reductions in dose and duration of chemotherapy regimens, potentially compromising clinical efficacy. In fact, aggressive chemotherapy has achieved remission in most types of cancers [2] but its use is often limited or even prohibited in elderly patients because of its cardiotoxicity. In haematological malignancies, anthracyclinsbased chemotherapy has represented the greatest risk for development of cardiotoxicity in this setting of patients.

The advent of new categories of drugs, like tyrosine-kinase inhibitors, proteosome-inhibitors or monoclonal antibodies has revolutionized the treatment of many blood cancers, such as chronic myeloid leukaemia, lymphoma, myeloma and others. However, although targeted therapies are usually better tolerated than chemotherapy, certain cardiac complications still remain and they can be very serious [3]; moreover, such new drugs are often used in combination with traditional chemotherapy.

Early identification of patients who are at risk for cardiotoxicity should be a primary goal for haematologists in the development of personalized antineoplastic therapeutic strategies or interventions.

Purpose of this article is to provide a comprehensive review of the adverse cardiac events associated with the use of the most common (chemo)-therapeutic agents used in haematological elderly patients not eligible for Stem Cell Transplantation (SCT), including new molecular-targeted drugs, and to provide guidance as how to prevent such effects.

*Address correspondence to this author at the Cattedra ed U. O. di Ematologia con trapianto, Azienda Ospedaliera Universitaria Policlinico di Palermo, Via del Vespro 129, 90127 Palermo; Tel: + 39091655 4419;

Fax: + 39091655 4402; E-mail: sergio.siragusa@unipa.It

\section{A. CHEMOTHERAPY FOR HAEMATOLOGICAL MALIG- NANCIES}

Haematological cancers represent a heterogeneous setting of diseases that can originate from highly indifferentiated to mature cells thus giving diseases different characteristics in terms of clinical presentation as well as response to therapy and prognosis. Despite these varieties, analogous chemotherapy has been proven efficacious in clinically different blood cancers that present a common origin. In Table 1 type and duration of the most common therapeutic protocol for treating acute and chronic myeloid and lymphoid neoplasm of adults are reported.

\section{ANTHRACYCLINES}

\section{Pathogenesis}

Anthracyclines have been extensively used for many years to treat a wide spectrum of haematologic malignancies and solid tumours [4]. The anthracyclines, doxorubicin and daunorubicin, are cytostatic antibiotics isolated from fungi belonging to the species Streptomyces. Anthracyclines, such as doxorubicin, and daunorubicin, continue to be some of the most active cytotoxic agents available and have broad activity against a variety of cancers, including acute myeloid and lymphoid leukaemias and solid tumours, such as lymphoma, sarcoma and breast cancer [5-7]. Daunorubicin has demonstrated activity against acute lymphocytic leukaemia and acute myeloid leukaemia.

Anthracycline-induced cardiotoxicity is categorised by the time of presentation as acute, early-onset or late-onset $[8,9]$. In acute anthracycline cardiotoxicity, symptoms manifest within hours or days of administration, often presenting as disturbances in intracardiac conduction and arrhythmias $[10,11]$. One of the best predictors of cardiotoxicity is the total cumulative dose of anthracycline $[12,13]$; generally, doses of $>400 \mathrm{mg} / \mathrm{m}^{2}$ are associated with the greatest risk of cardiac injury. The incidence of doxorubicininduced early $\mathrm{HF}$ is almost $3 \%$ at a cumulative dose of $400 \mathrm{mg} / \mathrm{m}^{2}$, $7 \%$ at a dose of $550 \mathrm{mg} / \mathrm{m} 2$ and $18 \%$ at $700 \mathrm{mg} / \mathrm{m} 2[14,15]$. In addition to the total cumulative dose, the incidence of acute and chronic cardiotoxicity may depend on the rate of anthracycline administration during each session [16], the schedule of delivery [17], the type of anthracycline used [18] and additional underlying risk factors, such as younger age in children and an age of $>65$ 
Table 1. Cardiac Toxicity and Proposed Prevention for the Most Commonly Used Chemo and Biological Therapy in Blood Cancers

\begin{tabular}{|c|c|c|c|}
\hline Type of Blood Cancer & Protocols' Acromynous & Type of Cardiotoxicity & Prevention of Cardiotoxicity \\
\hline Acute Myeloid Leukemia & $3+7$ & congestive heart failure & cardioprotective agents in conjunction with anthracyclines \\
\hline Acute Lymphoid Leukemia & & congestive heart failure & cardioprotective agents in conjunction with anthracyclines \\
\hline Chronic Myeloid Leukemia & Imatinib-dasatinb-nilotinib & $\begin{array}{l}\text { Oedema, dyspnea, left ventricu- } \\
\text { lar contractile dysfunction }\end{array}$ & Careful cardiac monitoring \\
\hline \multirow[t]{3}{*}{ Non Hodgkin Lymphomas } & R-CHOP & $\begin{array}{l}\text { supraventricular arytmia, tachy- } \\
\text { cardia, complete atrioventricular } \\
\text { block }\end{array}$ & Careful cardiac monitoring \\
\hline & R-CVP & $\begin{array}{c}\text { supraventricular arytmia, tachy- } \\
\text { cardia, complete atrioventricular } \\
\text { bloc }\end{array}$ & Careful cardiac monitoring \\
\hline & R-ICE & $\begin{array}{c}\text { supraventricular arytmia, tachy- } \\
\text { cardia }\end{array}$ & Careful cardiac monitoring \\
\hline Chronic Lymphatic Leukemia & FCR & $\begin{array}{l}\text { Acute onset heart failure and/or } \\
\text { myocarditis }\end{array}$ & Diuretics, angiotensin-converting enzyme inhibitor \\
\hline Hodgking lymphoma & ABVD & Acute onset heart failure & Use of liposomal encapsulation \\
\hline \multirow[t]{5}{*}{ Myeloma } & MP & bradycardia & \\
\hline & MP-THAL & bradycardia & \\
\hline & MPV & bradycardia & \\
\hline & THAL-DEX & bradycardia & \\
\hline & LEN & atrial fibrillation & \\
\hline
\end{tabular}

3+7: Cytarabine $100 \mathrm{mg} / \mathrm{m}^{2}$ i. v., Daunorubicin $45 \mathrm{mg} / \mathrm{m}^{2}$ i. v.

R-CHOP: Rituximab $375 \mathrm{mg} / \mathrm{m}^{2}$ i. v. , - Cyclophosphamide $750 \mathrm{mg} / \mathrm{m}^{2}$ i. v. - Adriamycin $50 \mathrm{mg} / \mathrm{m}^{2}$ i. v. ; Prednisone $100 \mathrm{mg} / \mathrm{m}^{2}$ oral

R-CVP: Rituximab $375 \mathrm{mg} / \mathrm{m}^{2} \mathrm{EV}$; Cyclophosphamide $750 \mathrm{mg} / \mathrm{m}^{2} \mathrm{EV}$; Vincristine $1.4 \mathrm{mg} / \mathrm{m}^{2}$ i. v. ,

Prednisone $100 \mathrm{mg} / \mathrm{m}^{2}$ oral

R-ICE: Rituximab $375 \mathrm{mg} / \mathrm{m}^{2}$ i. v. ; Ifosfamide $1 \mathrm{gr} / \mathrm{m}^{2}(\mathrm{dmax} 1,75 \mathrm{~g})$ i. v. ; Cisplatin $25 \mathrm{mg} / \mathrm{m}^{2}$ i. v. ; Etoposide $100 \mathrm{mg} / \mathrm{m}^{2}$ i. v.

FCR: Rituximab $375 \mathrm{mg} / \mathrm{m}^{2}$ (day 1); Fludarabine $25 \mathrm{mg} / \mathrm{m}^{2}$; Cyclophosphamide $750 \mathrm{mg} / \mathrm{m}^{2}$

ABVD: Adriamycin $25 \mathrm{mg} / \mathrm{m}^{2}$ i. v. ; Bleomycin $10 \mathrm{U} / \mathrm{m}^{2} \mathrm{EV}$; Vinblastine $6 \mathrm{mg} / \mathrm{m}^{2}$ i. v. ; Dacarbazine $375 \mathrm{mg} / \mathrm{m}^{2}$ i. v.

LEN: lenalidomide

MP: Melphalan $10 \mathrm{mg} / \mathrm{m}^{2}$ oral; Prednisone $60 \mathrm{mg} / \mathrm{m}^{2}$ oral

MP/Thal: Melphalan $10 \mathrm{mg} / \mathrm{m}^{2}$ oral; Prednisone $60 \mathrm{mg} / \mathrm{m}^{2}$ oral; Thalidomide $50-100 \mathrm{mg}$ oral

MPV: Melphalan $9 \mathrm{mg} / \mathrm{m}^{2}$ oral; Prednisone $60 \mathrm{mg} / \mathrm{m}^{2}$ oral; Bortezomib 1, $3 \mathrm{mg} / \mathrm{m}^{2}$ i. v.

Thal/Dex: Thalidomide $200 \mathrm{mg}$; Dexamethasone $40 \mathrm{mg}$

years in adults, African-American ancestry, female sex and trisomy 21 [19].

The cause of anthracycline-induced cardiotoxicity is probably multifactorial. Free radical-mediated myocyte damage is one of the most thoroughly studied mechanisms by which anthracyclines have been proposed to cause cardiotoxicity [20,21]. Pathologic findings in chronic anthracycline cardiotoxicity include loss of myofibrils within myocytes, vascular and mitochondrial degeneration and interstitial fibrosis [22, 23]. As myocytes undergo apoptosis and their cardiac growth responses to increasing somatic growth in children become inadequate, the LV walls become thinner, and the remaining cells compensate by becoming hypertrophic to create an LV mass that is as close as possible to normal for body surface area. This compensation means that many anthracycline-treated survivors with abnormal LV structure and function by echocardiography may show only histologic evidence of individual cardiomyocyte hypertrophy [24]. Anthracycline damage to all cardiac structures may begin with the first anthracycline dose [25]. Cardiotoxicity that occurs soon after anthracycline administration may be a harbinger of later toxicity; relatively little data exist about late- onset anthracycline cardiotoxicity in long-term survivors of adult malignancy [24].

Various methods have been recommended for the monitoring of cardiotoxicity in oncology. Recently, the applicability of cardiac troponins and creatine kinase MB CK-MB mass has been investigated in this setting and the results of clinical studies are inconsistent [26]. Horacek et al. [27] suggest that GPBB could be a new promising marker for detection of anthracycline-related cardiotoxicity and probably superior to cardiac troponins.

\section{Doxorubicin}

The most commonly used anthracycline is doxorubicin. Doxorubicin has a broad spectrum activity against both haematological cancers and solid tumours [28]. Doxorubicin cardiotoxicity is one of the most serious side effects of the cyclophosphamide, doxorubicin, vincristine, and prednisone chemotherapy, regimen, especially among elderly patients. In the CEOP regimen, epirubicin was substituted for doxorubicin to reduce cardiotoxicity. It is used in the treatment of acute leukaemias, both lymphocytic and myelogenous, [29] non-Hodgkin's lymphoma, [30] and Hodgkin's disease [31]. Doxorubicin is also effective against nonhaematological tu- 
mours, e. g. osteogenic sarcoma, [32] Ewing's sarcoma and Wilm's tumour, [33] and breast carcinoma [34].

The risk of doxorubicin induced congestive heart failure (CHF) increases with the cumulative dose. Cardiotoxicity is reported in $14 \%$ to $49 \%$ of patients treated for lymphoma, and among patients with NHL, the risk of CHF increases with the patient's age and history of coronary heart disease, valvular heart disease, hypertension, diabetes, cigarette smoking, or obesity [35, 36].

Hershman et Colleogues [37] found that hypertension, a known risk factor for $\mathrm{CHF}$, increases the risk of doxorubicin-related cardiotoxicity. Authors also demonstrated that selection factors may appropriately contribute to the receipt of doxorubicin-based chemotherapy in the elderly. Although late cardiac toxicity is increased with both longer duration of doxorubicin therapy and known predictors of HD, such as advanced age, comorbid conditions, prior heart disease, and diabetes, these other risks did not seem to potentiate the cardiotoxic effects of doxorubicin in Elderly Patients With Diffuse B-Cell Non-Hodgkin's Lymphoma.

\section{How to Prevent Anthracyclines Cardiotoxicity}

To maximize the beneficial effects of anthracyclines, much effort has been spent devising new strategies to decrease or prevent their cardiotoxic effects. In addition to various dosing schedules aimed at lowering peak plasma concentrations, an alternative strategy has been liposomal encapsulation. The most commonly used formulation is pegylated liposomal doxorubicin (Doxil). Which has a reduced tendency to accumulate in myocardial cells, with a resultant decrease in cardiotoxicity. Unlike traditional doxorubicin, pegylated doxorubicin has lower concentrations of free drug in the bloodstream and limited concentration in myocardial cells [38].

The non-pegylated liposomal anthracycline formulation (Myocet) had at least three randomized controlled trials with promising cardioprotective results. Myocet improves the therapeutic index of doxorubicin by significantly reducing cardiotoxicity and grade 4 neutropenia and provides comparable antitumour efficacy, when used in combination with cyclophosphamide as first-line therapy for MBC [39].

In recent years, novel anthracycline analogues have been developed with the hope that they will be as effective as conventional anthracyclines, but without the risk of cardiotoxicity. Idarubicin, a synthetic derivative of daunorubicin, is more lipophilic and has a longer half-life than danorubicin [40]. It is more lipophilic and can be administered orally as well as intravenously. Its main metabolite, idarubicinol, is as active as the parent drug. Idarubicin is effective at treating lymphoid and myeloid leukaemias, non-Hodgkin's lymphoma and advanced breast cancer. Similar to epirubicin, idarubicin causes less cardiotoxicity than doxorubicin [41].

Another approach to preventing anthracycline-induced cardiotoxicity is to use cardioprotective agents in conjunction with anthracyclines to mitigate their cardiotoxic effects. Dexrazoxane (ICRF-187) is a cardioprotective agent approved by the FDA. It is a bispiperazine, a nonpolar derivative of ethylenediamintetraacetic acid and a water-soluble positive enantiomer of the racemic drug razoxane. The suggestion that Fe plays an important role in anthracycline cardiotoxicity has been strengthened by observation that the chelator, dexrazoxane (ICRF-187), has a potent cardioprotective effect [42]. Despite the beneficial effects of dexrazoxane, concerns exist about the lack of information on its effect on late onset progressive cardiomyopathy, the lack of conclusive evidence that it reduces overall morbidity and mortality in paediatric patients, and its possible interference with the antitumour efficacy of anthracyclines [39].

\section{ALKYLATING AGENTS}

\section{Cyclophosphamide}

Cyclophosphamide (CY) is a non-cell-cycle-specific alkylating agent. It is a broadly active antineoplastic and immunosuppressant agent used in combination chemotherapy for non-Hodgkin's lymphoma, leukaemia, Hodgkin's disease, Burkitt's lymphoma, multiple myeloma, endometrial cancer, lung cancer and breast cancer [2]. Cardiotoxicity due to CY was reported in 1972 as a complication of bone marrow transplantation [43]. CY causes a direct damage to the vascular endothelium, followed by transudation of the toxic metabolite and the resulting damage to myocytes, interstitial haemorrhage, odema, and intercapillary microthrombi responsible for ischaemic myocardial damage [44]. Cardiac toxicity caused by CY seems to be due to pharmacokinetics and metabolism of the drug showing tenfold interindividual variation, most probably because of the impact of genetic polymorphism on the activity of liver microsomal enzymes [39]. In contrast to doxorubicin-induced cardiotoxicity, high-dose CY causes acute cardiac dysfunction that is independent of cumulative dose. CY doses greater than $1.5 \mathrm{~g} / \mathrm{m} 2$ may be associated with cardiotoxic risk [40]. Cardiac toxicity is manifested by acute on set heart failure and/or myocarditis occurring within 1-10 days after the administration of the first dose of CY. Some reports indicated that prior radiation therapy to the mediastinum or chest wall is an independent predictor of cardiotoxicity in patients with lymphoma undergoing high-dose chemotherapy [45]. A total dose ranging from 180 to $200 \mathrm{mg} / \mathrm{kg}$ over 2 to 4 days has been reported to cause symptomatic cardiomyopathy [46]. However, a dose as low as $120 \mathrm{mg} / \mathrm{kg}$ may also result in cardiotoxicity.

Goldberg et al, found that doses based on body surface area rather than body weight correlate well with incidence of cyclophosphamide-induced cardiotoxicity [47]. The fact that young children have a relatively higher body surface area probably explains the lower incidence and severity of cyclophosphamide-induced cardiotoxicity in them compared to adolescents and adults [47]. Several widely employed combinations of High-Dose (HD) cyclophosphamide are not associated with an increased risk of cardiotoxicity over single-agent HD cyclophosphamide. However, the risk for HD cyclophosphamide- associated cardiac toxicity may be increased by the concomitant administration of cytarabine or mitoxantrone [48].

Very little is known about cardiac toxicity of the treatment regimen with $\mathrm{CY}$ as part of stem cell collection followed by two autologous HSCT treatments with myeloablative dosages of MEL given in a very short time. Samo et al. [49] showed that MM patients treated with tandem autologous HSCT develop transient, mostly reversible and clinically non-overt neurohormonal activation of heart failure in each phase of treatment.

\section{How to Prevent Cyclofosfamide Cardiotoxicity}

Although there are no specific predictive parameters of cardiotoxicity, cardiac monitoring in high-risk patients undergoing high dose CY therapy may help to prevent serious cardiotoxicity [50]. It has been reported that cyclosporin-A, administered with CY, prevents cardiotoxicity [30], suggesting that CY induced cardiotoxicity is associated with mitochondrial membrane permeability to $\mathrm{Ca} 2+$ and that cyclosporine-A may restore mitochondrial permeability and prevent apoptosis of cardiomyocytes[51, 52]. Therapy with diuretics should be started in the first instance. The addition of an angiotensin-converting enzyme inhibitor in case of ECG and/or two-dimensional echocardiogram (2D echo) evidence of impaired left ventricular contraction, the patient not being hypotensive, should be considered according to established guidelines [53]. 


\section{B. NON CHEMOTHERAPIC AGENTS}

\section{Tyrosin-Kinase Inhibitors (Imatinib, Dasatinib and Nilotinib)}

Imatinib, an example of a molecular-targeted drug, has revolutionised treatment of chronic myeloid leukaemia (CML), and is now recommended as standard treatment for the accelerated and chronic-phase disease [54]. The cardiac effect of imatinib is controversial $[54,55]$. Cardiac toxicity may range from asymptomatic subclinical abnormalities such as electrocardiographic changes and left ventricular ejection fraction decline to life threatening events like congestive heart failure and acute coronary syndrome.

Clinical trials of the agent have reported a relatively high incidence of peripheral odema (63-66\%), some of which has been classified as severe (4-5\%). In addition, dyspnea has been reported in $12-16 \%$ of treated individuals and has been classified as severe in 4-5\% [56]. Cardiomyocytes from subjects who are being treated with imatinib appear to have endoplasmic reticula that have been activated in response to cellular stress, collapsed mitochondrial membrane potential, reduced ATP content, and to be more prone to cell death [56]. However, whether the observed cardiomyocyte cell damage represents clinical cardiotoxicity is still controversial [57], as is the role of the protooncogene abl (a target of imatinib and similar compounds) in the physiology of the cardiomyocyte [56, 57].

Kerkela et al. [58] report ten individuals who developed severe congestive heart failure while on imatinib and they show that imatinib-treated mice develop left ventricular contractile dysfunction. With imatinib treatment, cardiomyocytes in culture show activation of the endoplasmic reticulum (ER) stress response, collapse of the mitochondrial membrane potential, release of cytochrome $c$ into the cytosol, reduction in cellular ATP content and cell death.

It has been reported that individuals treated with imatinib developed severe CHF due to myocyte contractile dysfunction [58]. All patients had their left ventricular ejection fraction (LVEF) calculated by radionuclide imaging before the onset of treatment and after they developed symptoms of heart failure. In a series of 103 patients with CML treated with imatinib[59] and 57 patients with CML not treated with imatinib, no statistical difference was observed between the two groups regarding cardiac symptoms and signs, BNP levels, and echocardiographic measurements. However, peripheral oedema was more frequent in the group that received imatinib.

The early detection and application of the treatment for left ventricular dysfunction is of key importance for the prevention of irreversible myocardial injury. Careful cardiac monitoring and assessment by a cardiologist throughout the course of treatment with those TKIs that exert cardiac toxic effect is of primary importance [3].

Dasatinib is a multitargeted kinase inhibitor against Bcr-Abl, cKit, PDGFR-a and $b$ and the SRC family of kinases. Although clinical trials report high rates of peripheral oedema [60], only a $2 \%$ incidence of congestive heart failure as well as arrhythmias (including tachycardia) has been associated with Dasatinib treatment [61]. Nilotinib is an inhibitor of Bcr-Abl, c-Kit and PDGFRa and b receptors; Except for QT prolongation on ECG, no other cardiac event has been reported [62].

\section{RITUXIMAB}

Rituximab is a recombinant, chimeric anti-CD20 antibody that has emerged in recent years as an effective therapy for nonHodgkin's lymphoma (NHL) and other B-cell malignancies [63]. Rituximab binds to the CD20 antigen expressed on almost all malignant B-cells and leads to their destruction; it is used usually in combination of various protocols of chemotherapy. Treatment with rituximab is generally well tolerated and can be combined with chemotherapy, improving response rate, response duration, and in some cases overall survival of patients with B-cell lymphomas.
The cardiotoxicity of Rituximab has been reported when administered with chemotherapy or as monotherapy. Cardiac complications involving the conduction system, such as supraventricular arrhythmia and tachycardia, frequently occur in rituximab/ CHOP therapy [64]. In these cases, it may be difficult to correctly determine which medication, rituximab or anthracycline, cause them. Some authors reported a frequency of cardiac toxicity, manifested as grade 3 and 4 arrhythmias and supraventricular arrhythmias (such as tachycardia and auricular fibrillation or flutter) as high as 5. 9\% when rituximab is used with CHOP (cyclophosphamide, doxorubicin, oncovin, and prednisone) versus a level of $1.0 \%$ in patients who received chemotherapy alone [64]. During events of heart failure, higher levels of tumour necrosis factor- $\alpha$ (TNF- $\alpha$ ) and interleukin- 6 (IL-6) were observed, though this association might be related to a greater extent to their vasodilating compensating effect, unable to repair the endothelial damage seen in these situations [65].

Other clinical experiences reported unusual cardiac toxicity during rituximab treatment. Cervera et al. [66] described a case of elderly woman treated with Rituximab in monotherapy who experienced a complete atrioventricular block (CAVB) after the fifth administration. Authors explained such event likely due to cytokine release by anti-CD 20. Arai Y et al. [67] report herein a patient with mantle cell lymphoma (MCL) presenting ventricular tachycardia during infusion of rituximab. He received rituximab intravenously at a dose of $375 \mathrm{mg} / \mathrm{m} 2$ weekly for eight consecutive courses. During the rituximab infusion, electrocardiogram monitoring was performed. The second to seventh courses were eventless, although the first course was associated with pyrexia. However, transient ventricular tachycardia occurred during the infusion of the eighth course. Foran reported that $7 \%$ of 131 patients with MCL, immunocytoma, or small B-cell lymphocytic lymphoma who received rituximab as a single agent developed arrhythmia, including bradycardia, atrial fibrillation, tachycardia, and premature ventricular complexes [68]. Ponterucha et al. [69] (reported the 1st case report of symptomatic polymorphic ventricular tachycardia (VT) which occurred during an initial infusion of rituximab.

However, such findings were not reported by others. Kilickap et $a l$. [70] evaluated whether the addition of rituximab to an anthracycline-based regimen (CHOP) resulted in an increase in subclinical cardiac toxicity. Patients who were to receive $\mathrm{CHOP}$ or rituximab plus $\mathrm{CHOP}$ (R-CHOP) combination chemotherapy with a diagnosis of NHL were included in the study. Diastolic and systolic cardiac parameters, by using two-dimensional echocardiography, conventional pulsed-wave Doppler echocardiography and tissue Doppler echocardiography, were monitored before and after chemotherapy. There were 28 (M/F; 14/14) patients on CHOP and 33 (M/F; 16/17) patients on R-CHOP; Parameters of systolic function such as LVEF and FS did not significantly change in any patient. Doppler parameters of diastolic function such as lateral $\mathrm{E}$ and septal $\mathrm{E}$ velocity of mitral annulus decreased significantly after therapy $(\mathrm{P}<0.001)$. However, the decrease in diastolic function was similar in both arms $(\mathrm{P}>0.05)$. The addition of rituximab to CHOP chemotherapy does not significantly increase the risk of doxorubicin induced cardiotoxicity during administration and in the follow-up. Siano M. et colleagues have shown that the administration of rituximab with a maximal dose of $700 \mathrm{mg}$ over 60 minutes without steroid premedication is feasible and safe, provided one well-tolerated rituximab dose had been given in the previous 3 months. Patients with any type of B-cell lymphoma were eligible; Cohorts of at least three patients were assigned to rituximab with or without concomitant chemotherapy. An extensive cardiologic evaluation was done concomitantly to evaluate whether the increased infusion rate induced cardiotoxicity. Only one patient showed totally asymptomatic ECG alterations (repolarization disorder) of still uncertain origin. This alteration was correlated to rituximab infusion-although a coronary heart disease was not formally ruled out - and after a reduction 
of the infusion rate, no further ECG alterations were reported. In all cases, normal troponin values along with unchanged echocardiograms and Holter ECGs confirmed the lack of cardiotoxic effect of a fast infusion rate. Zaja et al. [71] investigate rituximab efficacy in previously untreated adult ITP patients with a platelet count of $20 \mathrm{x}$ $10(9) / \mathrm{L}$ or less. One hundred three patients were randomly assigned to receive $40 \mathrm{mg} / \mathrm{d}$ dexamethasone for 4 days with or without 375 $\mathrm{mg} / \mathrm{m} 2$ rituximab weekly for 4 weeks. One patient experienced supraventricular tachycardia during the first administration of rituximab, and 1 patient experienced seizure during the salvage treatment: both were probably related to study drug, and both patients were discontinued from the study. One patient with a previous history of vascular disease had a transitory ischaemic attack not related to study drug during salvage therapy.

\section{PROTEASOME INHIBITORS}

Bortezomib (Velcade, formerly PS-341) is a selective proteasome inhibitor that shows strong activity in vitro and in vivo against solid and haematologic malignancies [72]. Preclinical study results show that bortezomib suppresses tumour cell growth, induces apoptosis, overcomes resistance to standard chemotherapy agents and radiation therapy, and inhibits angiogenesis. Phase I study results established the antitumour activity of bortezomib, administered alone or in combination with standard chemotherapy agents, in patients with advanced haematologic malignancies or solid tumours, usually without additive toxicities [73].

Enrico $\mathrm{O}$ et al. [74] reported cardiotoxicity in haematological patients treated with bortezomib. Eight (11.6\%) of 69 patients developed serious cardiac side effects requiring medication, hospitalisation or an implant of a pacemaker. All eight patients who experienced cardiotoxicity underwent at least four cycles of bortezomib, strengthening the hypothesis of a direct connection with the drug. Authors explained that bortezomib may simultaneously cause atherosclerotic plaque progression and tendency to rupture, and facilitate ischaemic heart complications by reducing/abrogating myocardial preconditioning.

Takamatsu et al. [75] reported a case of a 79-year-old female patient with multiple myeloma who had no prior cardiac disease history developed an acute myocardial infarction on day 5 after receiving bortezomib and dexamethasone (BD). After treatment of coronary stenoses by stents, she received another course of BD therapy and developed angina pectoris on day 5 after the therapy.

Additionally, there is much experimental evidence to support the crucial role of a deficient proteasome activity in impairing cardiac function by several mechanisms, the most important of which is putatively the accumulation of unfolded, damaged and undegraded proteins inside myocytes (as extensively reviewed by Willis \& Patterson, 2006).

Zheng Hu et al. [76] tested the therapeutic efficacies as well as adverse effects of low dose imatinib mesyllate (IM) in combination with proteasome inhibitor, Bortezomib (BOR) or proteasome inhibitor I (PSI), in two CML murine models, and investigated possible mechanisms of action on chronic myeloid leukaemia (CML) cells. Their results demonstrated that low dose IM in combination with BOR exerted satisfactory efficacy in prolongation of life span and inhibition of tumour growth in mice, and did not cause cardiotoxicity or body weight loss.

\section{ANTIANGIOGENETIC DRUGS}

\section{Thalidomide, Lenalidomide, Pomalidomide}

Angiogenesis is a vital process resulting in the formation of new blood vessels [77]. While several studies have found moderate improvements when treated with angiogenesis inhibitors, greater success is being seen when the inhibition of angiogenesis is combined with other traditional forms of available therapy [78]. Thalidomide, an immunomodulatory and antiangiogenic agent, is useful in the treatment of some haematologic (mainly myeloma and other plasma-cell related disorders) and oncologic diseases. Thalidomidemechanisms of action are multifactorial based on immunomodulation, antiangiogenesis and cytokines regulation, particularly tumour necrosis factor-alpha. It has been reported that up to $6.8 \%$ of thalidomide-treated patients present bradycardia [79].

Lopez-de la cruz et al. [78] reported their experience with Thalidomide in patients with haematologic diseases. In a 34-month period, 33 patients with different haematologic diseases (multiple myeloma [MM], 20; myelodysplastic syndrome, eight; Waldenström macroglobulinemia, two; non-Hodgkin's lymphoma, two; malignant histiocytosis, one) were treated with thalidomide. Of them, five (15.1\%) had bradycardia, all with MM. Bradycardia was detected with a daily thalidomide dose ranging from 100 to $300 \mathrm{mg}$ and the time patients received thalidomide before cardiac event went from one to 18 months. Palladini et al. [79] evaluated the combination of thalidomide and intermediate-dose dexamethasone (T-Dex) in 31 patients with AL who did not respond to, or whose disease relapsed after, first-line therapy. In this study, symptomatic bradycardia emerged as a common (26\%) adverse reaction to thalidomide in patients with AL. Bradycardia was reported in 1 of 16 patients by the Boston group [80]) and in 1 of 12 patients by the Mayo Clinic Group [81]. A recent retrospective study performed on 96 myeloma patients treated with thalidomide showed a decrease in heart rate below 60 beats/min in $53 \%$ of patients and symptomatic bradycardia in $19 \%$ of patients, 23 which initially went unrecognized because studying the heart rate was not within the objectives of the original Trial [82].

Interestingly, despite these findings, few studies suggested the role for thalidomide in the management of chronic heart failure (CHF) in addition to traditional cardiovascular medications. In fact, Thalidomide is a drug with potential immunomodulating and matrix-stabilizing properties, that can improve left ventricular (LV) function in patients with CHF secondary to idiopathic dilated cardiomyopathy (IDCM) or coronary artery disease (CAD). Gullestad et al. [83] reported in their double-blind, placebo controlled study, that thalidomide increases LVEF and that these changes are accompanied by a decrease in LVEDV, suggesting that thalidomide can alleviate adverse myocardial remodelling and improve LV function in CHF. Importantly, this improvement in LV function occurred in the patients who were clinically stable and who, in the majority of cases, were receiving angiotensin-converting enzyme inhibitors or angiotensin II and_-blockers in addition to diuretics.

The use of thalidomide is limited by adverse effects of sedation, constipation, neuropathy and thromboembolism. In order to overpass such side-effects thus producing more potent and less toxic immunomodulators, thalidomide chemical structure has been modified and new compounds such as lenalidomide and pomalidomide were produced. Lenalidomide is approved by the US FDA for the treatment of patients with low-risk myelodysplastic syndrome (MDS) with deletion 5q cytogenetic abnormality [84]. Lenalidomide, unlike thalidomide, does not appear to cause peripheral neuropathy, but it does provoke cardiac arrhythmia such as atrial fibrillation (18\% versus $11 \%$ with placebo) and had severe haematological effects [85]. In two randomised, doubleblind, multicenter studies, the combination of lenalidomide and dexamethasone (LD) was compared with placebo and dexamethasone (PD) in patients with MM who had received at least one prior therapy [86]. The most clinically relevant grade 3 and 4 adverse events that occurred more frequently in the LD arm were neutropenia, thrombocytopenia, deep vein thrombosis, pulmonary embolism, and atrial fibrillation.

Immunomudulatory drugs (IMiDs) including thalidomide and lenalidomide have a proven role in the treatment of multiple myeloma (MM) and have significant activity in myelofibrosis (MF). Pomalidomide is the newest IMiD and appears to be promising for use in both MM and MF. IMiDs are hypothesised to act through multiple mechanisms including effects on angiogenesis and cyto- 
kine production [87]. Pomalidomide (POM) is an IMID significantly more potent than thalidomide, but without the limiting toxicities of neuropathy or sedation and cardiotoxicity.

\section{CONCLUSIONS}

Introduction of high-dose (HD) chemotherapy has improved the clinical outcome of patients with chemosensitive tumours, such as non-Hodgkin's lymphoma, Hodgkin's disease, multiple myeloma and leukaemias [88, 89]. However, cardiac toxicity has been frequently reported, particularly in HD cyclophosphamide-containing regimens, thus limiting their use especially in older patients.

Management of cardiac toxicity of chemotherapeutic agents is crucial since the increasing pool of long-term haematological cancer survivors. Preventative strategies that have met with some success have included the use of less cardiotoxic analogues such as epirubicin and liposomal anthracycline preparations. The role of investigational markers of myocardial injury, such as troponin $\mathrm{T}$ or brain natriuretic peptide, remains of great interest but still without robust evidences. In table 1 cardiotoxicity and its prevention in the most common antineoplastic regimens are reported.

The development of the so-called "targeted therapies", particularly drugs that inhibit the activity of tyrosine kinases, has become a remarkable progress in the treatment of neoplastic diseases. Recent data has shown that some of these therapies are associated with certain cardiotoxicity ranging from asymptomatic mild left ventricular dysfunction to congestive heart failure through different mechanisms.

In conclusion, old and new therapies for managing haematological malignancies are associated with cardiotoxicity; knowledge of any potential effect on cardiac function is crucial for the choice of the best regimen specially in elderly patients.

\section{REFERENCES}

[1] Gharib MI, Burnett AH. Chemotherapy-induced cardiotoxicity: current practice and prospects of prophylaxis. Eur J Heart Failure 2002; 235-42.

[2] Vinita BP, Milap CN. Cardiotoxicity of chemotherapeutic agents incidence, treatment and prevention. Drug Safety 2000; 22 (4): 263-302

[3] Orphanos GS, Ioannidis GN, Ardavanis AG. Cardiotoxicity induced by tyrosine kinase inhibitors. Acta Oncologica 2009; 48: 964-70

[4] Blum RH, Carter SK. Adriamycin. A new anticancer drug with significant clinical activity. Ann Intern Med 1974; 80(2): 249-59.

[5] Hinkle AS. Cardiotoxicity caused by chemotherapy. In: Late Effects of Childhood Cancer. Wallace WHB, Green DM (Eds). Edward Arnold, London 2004: 85-100.

[6] Tisma Dupanovic S, Shaddy RE, Wernovsky G. Cancer therapyrelated cardiotoxicities. In: Pediatric heart failure. Boca Raton: Taylor \& Francis 2005; pp. 665-738.

[7] Alvarez IA. Late effects after treatment of childhood cancer. Curr Opin Pediatr 2007; 19(1): 23-31.

[8] Friedman DL, Freyer DR, Levitt GA. Models of care for survivors of childhood cancer. Pediatr Blood Cancer 2006; 46(2): 159-68.

[9] Oeffinger KC, Wallace WH. Barriers to follow-up care of survivors in the United States and the United Kingdom. Pediatr Blood Cancer 2006; 46(2): 135-42.

[10] Hawkins MM, Robinson LL. Importance of clinical and epidemiological research in defining the long-term clinical care of pediatric cancer survivors. Pediatr Blood Cancer 2006; 46(2): 174-8.

[11] Ginsberg JP, Hobbie WL, Carlson CA, Meadows AT. Delivering long-term follow-up care to pediatric cancer survivors: transitional care issues. Pediatr Blood Cancer 2006; 46(2): 169-73

[12] Von Hoff DD, Layard MW, Basa P. Risk factors for doxorubicininduced congestive heart failure. Ann Intern Med 1979; 91(5): 7107.

[13] Swain SM, Whaley FS, Ewer MS. Congestive heart failure in patients treated with doxorubicin: a retrospective analysis of three trials. Cancer 2003; 97(11): 2869-79.

[14] Hortobagyi GM, Gutterman JU, Blumenschein GR, et al. Combination chemoimmunotherapy of metastatic breast cancer with 5- fluorouracil, adriamycin, cyclophosphamide, and BCG. Cancer 1979; 44: 1955-62.

[15] O'Bryan RM, Luce JK, Talley RW. Phase II evaluation of adriamycin in human neoplasia. Cancer 1973; 32: 1-8.

[16] Fumoleau P. Long-term cardiac toxicity after adjuvant epirubicinbased chemotherapy in early breast cancer: French Adjuvant StudyGroup results. Ann Oncol 2006; 17(1): 85-92.

[17] Owan TE, Hodge DO, Herges RM, Jacobsen SJ, Roger VL, Redfield MM. Trends in prevalence and outcome of heart failure with preserved ejection fraction. N Engl J Med 2006; 355: 251-9.

[18] Van Dalen EC. Different anthracycline derivates for reducing cardiotoxicity in cancer patients. Cochrane Database Syst Rev 2006; 4: CD005006.

[19] Simbre VC II. Cardiotoxicity of cancer chemotherapy: implications for children. Pediatr Drugs 2005; 7(3): 187-202

[20] Doroshow JH. Effect of anthracycline antibiotics on oxygen radical formation in rat heart. Cancer Res 1983; 43: 460-72

[21] Unverferth DV, Fertel RH, Balcerzak SP, Magorien RD, O'Dirisio MS. N-Acetylcysteine prevents the doxorubicin-induced decrease of cyclic GMP. Semin Oncol 1983; 10 (Suppl 1): 49-52

[22] Billigham ME. Anthracycline cardiomyopathy monitored by morphologic changes. Cancer Treat Rep 1978; 62(6): 865-72.

[23] Berry GJ, Jorden M. Pathology of radiation and anthracycline cardiotoxicity. Pediatr Blood Cancer 2005; 44(7): 630-7

[24] Barry E, Alvarez JA, Scully R, Miller T, Lipshultz SE. Anthracycline-induced cardiotoxicity: course, pathophysiology, prevention and management. Expert Opin Pharmacother 2007; 8(8): 1039-58

[25] Goldberg MA, Antin JH, Guinan EC. Cyclophosphamide cardiotoxicity: an analysis of dosing as a risk factor. Blood 1986; 68: 1114-8.

[26] Sparano JA, Brown DL, Wolff AC. Predicting cancer therapyinduced cardiotoxicity. The role of troponins and other markers. Drug Saf 2002; 25: 301-11.

[27] Horacek1 JM, Tichy M, Jebavy L, Pudil R, Ulrychova M, Maly Y. Use of multiple bionarkers for evaluation of anthracycline-induced cardiotoxicity in patients with acutemyeloid leukemia. Exp Otoxicitync 2008; 30(2): 157-9.

[28] Blum RH, Carter SK. Adriamycin: a new anticancer drug with significant clinical activity. Ann Intern Med 1974; 80: 249-59

[29] McCredie KB, Hewlett JS, Kennedy A. SequentialAdriamycinAra-C (A-OAP) for remission induction (RI) of adult acute leukemia (AAL). Proc Am Assoc Cancer Res 1976; 17: 239

[30] Schein PS, DeVita JR VT, Hubbard S, et al. Bleomycin, adriamycin, cyclophosphamide, vincristine and prednisone (BACOP): combination chemotherapy in the treatment of advanced diffuse histiocytic lymphoma. Ann Intern Med 1976; 85: 417-22

[31] Bonnadonna G, DeLena M, Oslenghi C. Combination chemotherapy of advanced Hodgkins disease (HD) with a combination of Adriamycin (ADM), bleomycin (BLM), vinblastine (VBL), and imidazole carboxamide (DTIC) versus MOPP. Proc Am Assoc Cancer Res 1974; 360: 90 .

[32] Cortes EP, Holland JF, Wany JJ. Amputation and adriamycin in primary osteosarcoma. N Engl J Med 1974; 291: 998-1000.

[33] O'Bryan RM, Luce JK, Talley RW. Phase II evaluation of adriamycin in human neoplasia. Cancer 1973; 32: 1-8.

[34] Hortobagyi GM, Gutterman JU, Blumenschein GR, et al. Combination chemoimmunotherapy of metastatic breast cancer. Cancer 1979; 44: 1955-62.

[35] Limat S, Demesmay K, Voillat L, et al. Early cardiotoxicity of the $\mathrm{CHOP}$ regimen in aggressive non- Hodgkin's lymphoma. Ann Oncol 2003; 14: 277-281.

[36] He J, Ogden LG, Bazzano LA, et al. Risk factors for congestive heart failure in US men and women: NHANES I epidemiologic follow-up study. Arch Intern Med 2001; 161: 996-1002,

[37] Hershman DL, McBride RB, Eisenberger A, Tsai WY, Grann WR, Jacobson YS. Doxorubicin, cardiac risk factors, and cardiac toxicity in elderly patients with diffuse B-cell non-Hodgkin's lymphoma. J Clin Oncol 2008; 26(19): 3159-65.

[38] Ewer MS. Cardiac safety of liposomal anthracyclines. Semin Oncol 2004; 31(6 Suppl 13): 161-81.

[39] Batist G, Ramakrishnan G, Rao CS. Reduced cardiotoxicity and preserved antitumor efficacy of liposome-encapsulated doxorubicin and cyclophosphamide compared with conventional doxorubicin and cyclophosphamide in a randomized, multicenter trial of metastatic breast cancer. J Clin Onc 2001; 19(5): 1444-54. 
[40] Robert J, Rigal-Huguet F, Hurteloup P, et al. Comparative pharmacokinetic study of idarubicin and daunorubicin in leukemia patients. Hematol Oncol 1992; 10(2): 111-6.

[41] Martoni A, Piana E, Guaraldi M, et al. Comparative Phase II study of idarubicin versus doxorubicin in advanced breast cancer. Oncology 1990; 47(5): 427-32.

[42] Kwok JC, Richardson DR. The cardioprotective effect of the iron chelator dexrazoxane (ICRF-187) on anthracycline-mediated cardiotoxicity. Redox Rep 2000; 5(6): 317-24.

[43] Santos GW, Sensenbrenner LL, Burke PJ, et al. The use of cyclophosphamide for clinical marrow transplantation. Transplant Proc 1972; 4: 559-64.

[44] Gottdiender JS, Appelbaum FR, Ferrans VJ, Diesseroth A, Ziegler J. Cardiotoxicity associated with high dose cyclophosphamide therapy. Arch Intern Med 1981; 141: 758-63.

[45] Braverman AC, Antin JH, Plappert MT. Cyclophosphamide cardiotoxicity in bone marrow transplantation: a prospective evaluation of new dosing regimens. J Clin Oncol 1991; 9: 1215-33.

[46] Dow E, Schulman H, Agura E. Cyclophosphamide cardiac injury mimicking acute myocardial infarction. Bone Marrow Transplant 1993: 12: 169-72

[47] Goldberg MA, Antin JH, Guinan EC, Rappeport JM. Cyclophosphamide cardiotoxicity: an analysis of dosing as a risk factor. Blood 1986; 68: 1114-8.

[48] Peters WP, Ross M, Vredenburgh JJ, et al. High-dose chemotherapy and autologous bone marrow support as consolidation after standard-dose adjuvant therapy for high-risk primary breast cancer. J Clin Oncol 1993; 11: 1132-43

[49] Zver S, Zadnik V. Cardiac toxicity of high-dose cyclophosphamide and melphalan in patients with multiple myeloma treated with tandem autologous hematopoietic stem cell transplantation. Int J Hematol 2008; 88: 227-36

[50] Taniguchi I. Clinical significance of cyclophosphamide-induced cardiotoxicity. Intern Med 2005; 44(2): 89-90.

[51] Al-Nasser IA. In vivo prevention of cyclophosphamide-induced $\mathrm{Ca} 2+$ dependent damage of rat heart and liver mitochondria by cyclosporin A. Comp Biochem Physiol A Mol Integr Physiol 1998; 121: 209-14.

[52] Beranek JT. Apoptosis contributes to cyclophosphamide-induced cardiomyopathy. Bone Marrow Transplant 2002; 29: 91.

[53] ACC/AHA guidelines for the evaluation and the management of chronic heart failure in the adult: executive summary. A report of the American College of Cardiology/American Heart Association Task Force on Practice Guidelines. J Am Coll Cardiol 2001; 38: 2101-13.

[54] Hehlmann R, Hochhaus A, Baccarani M. Chronic myeloid leukaemia. Lancet 2007; 370(9584): 342-50

[55] Kerkela R, Grazette L, Yacobi R. Cardiotoxicity of the cancer therapeutic agent imatinib mesylate. Nat Med 2006; 12 (8): 908-16.

[56] Cohen MH. Approval summary for imatinib mesylate capsules in the treatment of chronic myelogenous leukemia. Clin Cancer Res 2002; 8: 935-42.

[57] Will Y, Dykens JA, Nadanaciva S, et al. Effect of the multitargeted tyrosine kinase inhibitors imatinib, dasatinib, sunitinib, and sorafenib on mitochondrial function in isolated rat heart mitochondria and H9c2 cells Toxicol Sci 2008; 106(1): 153-61

[58] Ribeiro AL, Marcolino MS, Bittencourt HN, et al. An evaluation of the cardiotoxicity of imatinib mesylate . Leuk Res 2008; 32(12): $1809-14$

[59] Park YH, Park HJ, Kim BS, et al. BNP as a marker of the heart failure in the treatment of imatinib mesylate. Cancer Lett 2006; 243: $16-22$.

[60] Kantarjian H, Pasquini R, Hamerschlak N, et al. Dasatinib or highdose imatinib for chronic-phase chronic myeloid leukemia after failure of first-line imatinib: a randomized phase 2 trial. Blood 2007; 109: 5143-50.

[61] Galinsky I, Buchanan S. Practical management of dasatinib for maximum patient benefit. Clin J Oncol Nurs 2009; 13(3): 329-35. Review.

[62] Quintas-Cardama A, Cortes J. Nilotinib therapy in chronic myelogenous leukemia. Drugs Today 2007; 43: 691-702

[63] Maloney DG, Grillo-Lo'pez AJ, White CA, et al. IDEC-C2B8 (rituximab) anti-CD20 monoclonal antibody therapy in patients with relapsed low-grade non-Hodgkin's lymphoma. Blood 1997; 90: $2188-95$
[64 Coiffier B, Lepage E, Briere J, et al. CHOP chemotherapy plus rituximab compared with CHOP alone in elderly patients with diffuse large B-cell lymphoma. N Engl J Med 2002; 346: 235-42.

[65] Coma-Canella I, Macias A, Varo N, Sanchez Ibarrola A. Neurohormones and cytokines in heart failure. Correlation with coronary flow reserve. Rev Esp Cardiol 2005 58: 1273-7

[66] Cervera Grau JM, Esquerdo Galiana G, Belso Candela A, et al. Complete atrioventricular block induced by rituximab in monotherapy in an aged patient with non-Hodgkin's diffuse large B-cell lymphoma Clin Transl Oncol 2008; 10(5): 298-9.

[67] Arai Y. Ventricular Tachycardia Associated With Infusion of Rituximab in Mantle Cell Lymphoma. Am J Hematol 2005; 78: 3169.

[68] Foran JM, Rohatiner AZS, Cunningham D, et al. European phase II study of rituximab (chimeric anti-CD20 monoclonal antibody) for patients with newly diagnosed mantle-cell lymphoma and previously treated mantle-cell lymphoma, immunocytoma, and small B-cell lymphocytic lymphoma. J Clin Oncol 2000; 18: 317-24.

[69] Poterucha JT, Westberg M, Nerheim P, Lovell JP. Rituximabinduced polymorphic ventricular tachycardia. Tex Heart Inst J 2010; 37(2): 218-20

[70] Kilickap S, Yavuz B, Aksoy S, et al. Addition of rituximab to chop does not increase the risk of cardiotoxicity in patients with nonHodgkin's lymphoma. Med Oncol 2008; 25(4): 437-42.

[71] Zaja F, Baccarani M. Dexamethasone plus rituximab yields higher sustained response rates than dexamethasone monotherapy in adults with primary immune thrombocytopenia. Blood 2010; 115(14): 2755-62.

[72] Montagut C. The proteasome: a novel target for anticancer therapy, Clin Trans Oncol 2006; 8(5): 313-7.

[73] Jung L. Discovery, Development, and clinical applications of bortezomib, Oncology 2004 Dec 90. Takamatsu H, Yamashita T, Kotani T, Sawazaki A, Okumura H, Nakao S Ischemic heart disease associated with bortezomib treatment combined with dexamethasone in a patient with multiple myeloma. Oncology 2004; 18(14 Suppl 11): 4-13

[74] Enrico O, Gabriele B, Nadia C, et al. Unexpected cardiotoxicity in haematological bortezomib treated patients. Br J Haematol 2007; 138(3): 396-7.

[75] Takamatsu H, Yamashita T, Kotani T, Sawazaki A, Okumura H, Nakao S. Ischemic heart disease associated with bortezomib treatment combined with dexamethasone in a patient with multiple myeloma. Int J Hematol 2010; 91(5): 903-6.

[76] Hu Z, Pan XF, Wu FQ, et al. Synergy between proteasome inhibitors and imatinib mesylate in chronic myeloid leukemia. PLoS One 2009; 4(7): e6257.

[77] Nussenbaum F, Herman IM. Tumor angiogenesis: insights and innovations. J Oncol 2010; 2010: 132641.

[78] López-de la Cruz I, Aguayo-González A, López-Karpovitch X Thalidomide-associated bradycardia in patients with hematologic diseases: a single institution experience. Rev Invest Clin 2006; 58(5): 424-31. Review

[79] Palladini G, Perfetti V, Perlini S, et al. The combination of thalidomide and intermediate-dose dexamethasone is an effective but toxic treatment for patients with primary amyloidosis (AL). Blood 2005; 105(7): 2949-51

[80] Seldin DC, Choufani EB, Dember LM. Tolerability and efficacy of thalidomide for the treatment of patients with light chain-associated (AL) amyloidosis. Clin Lymphoma 2003; 3: 241-6.

[81] Dispenzieri A, Lacy MQ, Rajkumar SV, et al. Poor tolerance of thalidomide in patients with primary systemic amyloidosis. Amyloid 2003; 10: 257-26

[82] Fahdi IE, Gaddam V, Saucedo JF, et al. Bradycardia during therapy for multiple myeloma with thalidomide. Am J Cardiol 2004; 93 : 1052-5.

[83] Gullestad L, Ueland T, Fjeld JG, et al. Effect of thalidomide on cardiac remodeling in chronic heart failure: results of a doubleblind, placebo-controlled study. Circulation 2005; 112(22): 340814 .

[84] Shah SR, Tran TM. Lenalidomide in myelodysplastic syndrome and multiple myeloma. Drugs 2007; 67 (13): 1869-81.

[85] Lenalidomide: new drug. Myeloma: many questions remain unanswered. Prescrire Int 2008; 17 (98); 230-2

[86] Hazarika M, Rock E, Williams G, Dagher R, Sridhara R, Booth B Lenalidomide in combination with dexamethasone for the treat- 
ment of multiple myeloma after one prior therapy. Oncologist 2008; 13(10): 1120-7.

[87] Lacy MQ, Rajkumar SV. Pomalidomide: a new IMiD with remarkable activity in both multiple myeloma and myelofibrosis. Am J Hematol 2010; 85(2): 95-6. Review.

Received: June 3, 2010
[88] Craddock C. Haemopoietic stem-cell transplantation: recent progress and future promise. Lancet Oncol 2000; 1: 227-234.

[89] Rossini F, Terruzzi E, Perego D, et al. Long-Term Follow-Up of Patients with Intermediate or High-Grade Non-Hodgkin Lymphoma Treated with a Combination of Cyclophosphamide, Epirubicin, Vincristine, and Prednisone. Cancer 2004; 100(2): 350-5. 